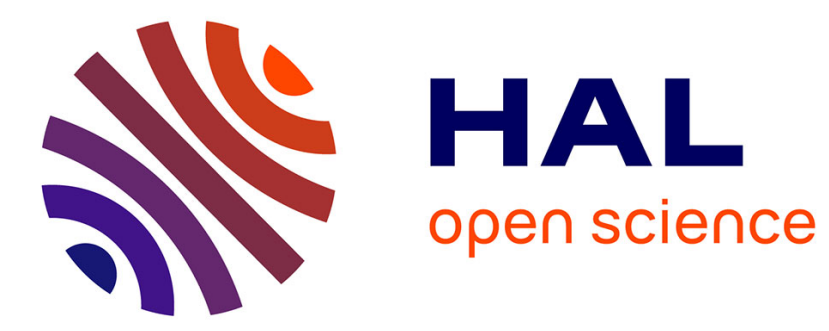

\title{
Elastic, thermal and structural properties of platinum
}

Shigeaki Ono, John P. Brodholt, G. David Price

\section{To cite this version:}

Shigeaki Ono, John P. Brodholt, G. David Price. Elastic, thermal and structural properties of platinum. Journal of Physics and Chemistry of Solids, 2011, 72 (3), pp.169. 10.1016/j.jpcs.2010.12.004 . hal-00720586

\section{HAL Id: hal-00720586 https://hal.science/hal-00720586}

Submitted on 25 Jul 2012

HAL is a multi-disciplinary open access archive for the deposit and dissemination of scientific research documents, whether they are published or not. The documents may come from teaching and research institutions in France or abroad, or from public or private research centers.
L'archive ouverte pluridisciplinaire HAL, est destinée au dépôt et à la diffusion de documents scientifiques de niveau recherche, publiés ou non, émanant des établissements d'enseignement et de recherche français ou étrangers, des laboratoires publics ou privés. 


\section{Author's Accepted Manuscript}

Elastic, thermal and structural properties of platinum

Shigeaki Ono, John P. Brodholt, G. David Price

PII: $\quad$ S0022-3697(10)00429-4

DOI:

Reference: doi:10.1016/j.jpcs.2010.12.004 PCS 6376

To appear in: Journal of Physics and Chemistry of Solids

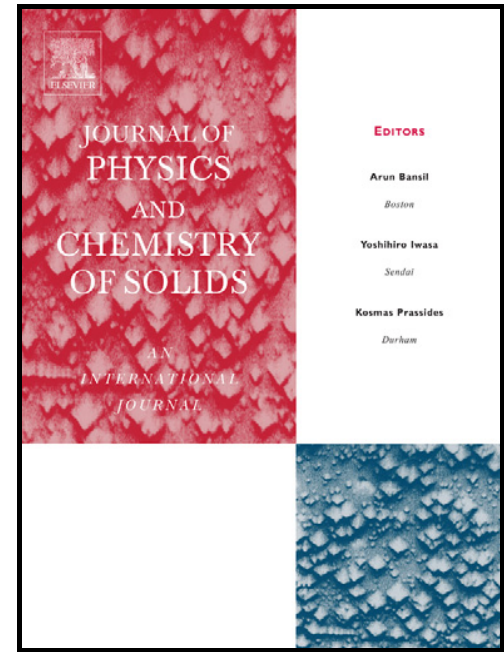

www.elsevier.com/locate/jpcs

Received date: 29 April 2010

Revised date: 5 October 2010

Accepted date: 3 December 2010

Cite this article as: Shigeaki Ono, John P. Brodholt and G. David Price, Elastic, thermal and structural properties of platinum, Journal of Physics and Chemistry of Solids, doi:10.1016/j.jpcs.2010.12.004

This is a PDF file of an unedited manuscript that has been accepted for publication. As a service to our customers we are providing this early version of the manuscript. The manuscript will undergo copyediting, typesetting, and review of the resulting galley proof before it is published in its final citable form. Please note that during the production process errors may be discovered which could affect the content, and all legal disclaimers that apply to the journal pertain. 


\section{Elastic, thermal and structural properties of platinum}

Shigeaki Ono, ${ }^{\text {a,b }}$ John P. Brodholt, ${ }^{\mathrm{a}}$ G. David Price ${ }^{\mathrm{a}}$

a Department of Earth Sciences, University College London, Gower Street, London WC1E 6BT, UK.

${ }^{\mathrm{b}}$ Institute for Research on Earth Evolution, Japan Agency for Marine-Earth Science and Technology, 2-15 Natsushima-cho, Yokosuka, Kanagawa 237-0061, Japan.

Corresponding author:

Shigeaki Ono

Institute for Research on Earth Evolution, Japan Agency for Marine-Earth Science and Technology, 2-15 Natsushima-cho, Yokosuka, Kanagawa 237-0061, Japan.

Fax: +81-(0)46-867-9625

Phone: +81-(0)46-867-9631

E-mail: sono@jamstec.go.jp 


\section{Abstract}

The thermal equation of state (EOS) for platinum has been calculated to 300 $\mathrm{GPa}$ and $3000 \mathrm{~K}$ using ab initio molecular dynamics employing the local density approximation (LDA) and the projector augmented-wave methods (PAW). Direct ab initio molecular dynamics avoids the simplifying assumptions inherent in empirical treatments of thermoelasticity. A third-order Birch-Murnaghan equation EOS fitted to the $300 \mathrm{~K}$ data yielded an isothermal bulk modulus of $B_{T 0}=290.8 \mathrm{GPa}$ and a pressure derivative of $B_{T}{ }^{\prime}=5.11$, which are in better agreement with the measured values than those obtained by previous calculations. The high-temperature data were fitted to a thermal pressure EOS and a Mie-Grüneisen-Debye EOS. The resulting calculated thermal expansion coefficient, $\alpha_{0}$, temperature derivative of the isothermal bulk modulus, $\left(\frac{\partial B_{T}}{\partial T}\right)_{V}$, and second temperature derivative of the pressure, $\left(\frac{\partial^{2} P}{\partial T^{2}}\right)_{V}$, were $1.94 \times 10^{-5} \mathrm{~K}^{-1},-0.0038 \mathrm{GPaK}^{-1}$, and $1.7 \times 10^{-7} \mathrm{GPa}^{2} \mathrm{~K}^{-2}$, respectively. A fit to the Mie-Grüneisen-Debye EOS yielded values for the Grüneisen parameter, $\gamma_{0}$, and its volume dependence parameter, $q$, of 2.18 and 
1.75, respectively. An analysis of our data revealed a strong volume dependence of the thermal pressure of platinum. We also present a qualitative analysis of the effects of intrinsic anharmonicity from the calculated Grüneisen parameter at high temperatures.

Keyword: Equation of state, Platinum, Ab initio calculations, High pressure and high temperature

\section{Introduction}

Significant technological advances have been made recently in static high pressure experiments. The $P-T$ range of experiments has been extended and accurate measurements can be performed under very high pressure and temperature conditions using, for example, third-generation synchrotron facilities. It is important to know accurate experimental pressures to minimize the uncertainty of experimental data. However, there is no direct way to measure the absolute pressure. Instead of direct pressure measurements, several secondary pressure scales can be used, such as luminescence scales [1,2] or 
equation of state (EOS) scales $[3,4]$. The ruby luminescence pressure scale is the most widely used pressure scale at ambient temperature. However, this scale cannot be used at high temperatures. EOS scales can be applied at high temperatures, and several EOSs for various internal calibrants have been proposed for use in in situ high-pressure and high-temperature experiments. Many materials have been used as internal pressure standards, including $\mathrm{NaCl}$ [4,5], $\mathrm{MgO}[6,7], \mathrm{Au}[3,8]$, and Pt [8,9]. Both $\mathrm{Au}$ and Pt are often used in laser-heated diamond anvil cell experiments at extremely high pressures because of their ability to absorb laser power and their chemical inertness [10-12]. The outcome of debates on an accurate pressure value of the seismic discontinuity in the Earth's mantle depends critically on the accuracy of the EOS employed [13,14]. According to these debates, present EOSs are not accurate enough for application to pressure-sensitive issues. However, efforts to establish a set of internally consistent pressure scales or to revise the EOS of various materials have been made [15-18]. However, no reliable pressure scales are presently widely accepted.

Thermoelastic parameters need to be measured to establish an EOS for 
a material. However, measured parameters often include a significant uncertainty value, and this amplifies the uncertainties in the inferred pressure when the EOS is extrapolated to extreme conditions. As a result, and because of the increasing need for accuracy of a method, it is desirable to complement experimentally determined EOSs with those inferred from quantum mechanical electronic structure calculations.

$A b$ initio calculations have been successfully used to predict the structural phase stability of crystalline materials over a wide range of $\mathrm{P}$ and $\mathrm{T}$. However, previous ab initio electronic structure calculations have not been able to predict pressure-temperature-volume relationships to the accuracy required for EOS standards, although such calculations have provided valuable qualitative guidance.

The EOS of $\mathrm{Pt}$, which has a face-centered cubic (fcc) structure, is commonly used as a pressure standard, because this phase is stable from ambient conditions to very high pressures and temperatures. The EOS of Pt is based on shock experimental data reported by Jamieson et al. [8]. Another EOS of $\mathrm{Pt}$ was based on a first-principle theoretical treatment combined with 
experimental data [9]. Although these EOSs have been commonly used as pressure standards in high-pressure experiments, the reliability of the Pt pressure standard has been questioned in a recent study [19]. As a result, several works based on the density functional theory (DFT) aimed at refining the Pt EOS have been reported [20-23]. However, these studies were all based on ground state calculations (i.e. at $0 \mathrm{~K}$ ).

In this study, ab initio molecular dynamics (AIMD) calculations were performed to establish the EOS for Pt to $300 \mathrm{GPa}$ and $3000 \mathrm{~K}$. Before the $a b$ initio molecular dynamics calculations were performed, static calculations were used to assess relativistic effects and the effects of different approximations of the exchange-correlation term used in our DFT calculations. All the AIMD calculations for Pt were carried out without spin-orbit coupling effects. As the results presented here are intended for use in extending the Pt pressure standard, extensive tables of results are provided.

\section{Methods}

The electronic structure of Pt was calculated using the projector 
augmented wave (PAW) implementation of the DFT using the Vienna ab initio simulation software package (VASP) [24-26]. The local density approximation (LDA) was used in the AIMD simulations for the exchange-correlation potential. We also used the PW91 [27] and the PBE96 [28] functionals of the generalized gradient approximation (GGA) in the static calculations to assess the influence of the different approximations on the EOS of Pt. The PAW method was used for the core electrons, and a core radius used for Pt was 2.5 a.u. in all cases. A plane-wave cut-off of $500 \mathrm{eV}$ was used in the static calculations. For the Brillouin zone sampling, we used a 12x12x12 Monkhorst-Pack mesh in a primitive unit cell, which provided the convergence of the total energy to within $1 \mathrm{meV} /$ atom. The calculations were performed at ten selected pressures in the pressure range 0-200 GPa.

In the AIMD simulations, we used two assumptions: (1) that the position and moment of the nuclei evolved in time, according to Newton's equations of motion; and (2) that the forces on the nuclei at any given time are produced by the electron sub-system in its ground state, calculated as though the nuclei were completely static at their current instantaneous positions (the Born-Oppenheimer approximation). We used a 64-atom supercell, with $\Gamma$-point Brillouin zone sampling, a time step of $1 \mathrm{fs}$, and a 
plane-wave cut-off of $300 \mathrm{eV}$ for the AIMD simulations at a constant volume. The simulations were run in the constant $N V T$ ensemble with a Nosé thermostat [29] for at least 3 ps after equilibration. The computation time required to reach equilibrium varied between configurations, and depended on the starting atomic position, velocity, temperature, and pressure. In previous studies, we have confirmed that useful data for the elastic properties of solids at high P-T conditions can be acquired using the AIMD calculations in previous studies [5,30,31]. In this study, our AIMD calculations were performed at 40 selected P-T conditions in the pressure and temperature ranges of 0-300 GPa and 300-3000 K, respectively. To assess the reliability of our method, the elastic properties of Pt were also calculated and compared with the elastic properties reported in previous experimental studies. A comprehensive description of our method as applied to the modeling of condensed matter has been given in a recent publication $[32]$.

\section{Test of methods at zero temperature}

We carried out three tests at zero temperature in the pressure range 0-200 GPa to assess the accuracy of our calculations. Our molecular dynamics 
calculations were based on the DFT. As the system evolves over time, the energy and the atomic forces at the atomic positions at any given instant were calculated using the DFT technique. Therefore, the uncertainty of the AIMD simulations was at least as great as that of the DFT calculations at zero temperature. There are three significant factors that need to be simulated accurately to determine a reliable EOS for Pt.

First, we tested the influence of spin-orbit coupling on the EOS of Pt using LDA calculations. We found that spin-orbit coupling had a small effect on the EOS. The influence of spin-orbit coupling on the $P-V$ relationship is shown in Fig. 1 up to a pressure of $200 \mathrm{GPa}$. The difference in volume was very small in the pressure range investigated. When our calculations included spin-orbit coupling, we observed an increase of about $0.2 \%$ in the equilibrium volume at ambient pressure (Table 1). This deference in volume decreases as the pressure increased (Fig. 1b). This small effect is in agreement with previous calculations on Pt carried out by Bercegeay and Bernard [20].

Second, we assessed the accuracy of different exchange-correlation functionals, which required us to assess the accuracy of the predicted 
zero-pressure density, which in turn led to the application of a simple pressure correction. In general, it is known that DFT calculations systematically either overestimate or underestimate the equilibrium volume of a solid. The LDA underestimates the volume by a few percent, whereas the GGA usually overestimates the volume. To correct for this error, a recent study [33] has shown that adding a simple, fixed pressure correction offset to the GGA calculated pressure lead to very good agreement between the calculated and experimental lattice parameters, bulk modulus, and elastic constants of a silicate material.

We calculated the equilibrium volumes using the LDA, GGAs of PW91 [27] and PBE96 [28] to assess the size and nature of this error for Pt. The general trends in our calculations were in agreement with previous studies on Pt (Table 1). The experimental values of the volume and bulk modulus for Pt show intermediate values between the LDA and the GGA results. This indicates that an effective correction is necessary for both the LDA and the GGA calculations to provide a reliable EOS of Pt.

To address this, we used the following simple pressure modification of:

$$
P_{\text {total }}(V, T)=P_{s t}(V, 0)+P_{t h}(V, T)+\Delta P,
$$


where $P_{\text {total }}(V, T)$ denotes the total pressure at volume $V$ and temperature $T$. The terms $P_{s t}(V, 0)$ and $P_{t h}(V, T)$ denote the static pressure at volume $V$ and $0 \mathrm{~K}$, and the thermal pressure at volume $V$ and temperature $T$, respectively. The simple constant shift in pressure $(\Delta P)$ brings the EOS into good agreement with the DFT calculations and the experimental data (Table 1). Finally, the value of $\Delta P$ in this study, which applies to temperatures in the range $300-3000 \mathrm{~K}$, was determined at $300 \mathrm{~K}$ using AIMD calculations.

We also assessed the effect of different exchange-correlation functionals on the thermal properties. The thermal pressure, $P_{t h}(V, T)$, was calculated at 0 and 300 GPa using AIMD calculations. Fig. 2 shows the difference in the thermal pressure of the GGA exchange-correlation functionals relative to LDA. For pressures of 0 and $300 \mathrm{GPa}$, the GGA functionals underestimated the thermal pressure relative to the LDA functional. However, the difference was very small compared with the uncertainty in the static pressure, $P_{s t}(V, 0)$.

After these tests, it was concluded that the use of the LDA approximation was adequate; therefore, the LDA approximation was used in the subsequent AIMD calculations. As the influence of spin-orbit coupling on 
the bulk modulus was $0.5 \%$ after the pressure correction was applied, and because we needed to save on computation time for the AIMD method, we decided to neglect spin-orbit interactions. The pressure correction was also applied to the AIMD simulations.

\section{Stability of the fec structure}

The hexagonal close-packed (hcp) structure is already known to be the most stable state for some metals at high pressures (e.g. Fe). Therefore, the stability of the face-centered cubic (fcc) structure of Pt at high pressures was investigated in this study. Calculations were performed in the static state (i.e., at $0 \mathrm{~K}$ ). The difference in enthalpy, which is the same as the Gibbs energy when the temperature is zero, was calculated directly from $H=E+P V$ to investigate the stability of each structure, where $E, P$, and $V$ are the internal energy, pressure, and volume, respectively.

Fig. 3 shows the stability of the fcc structure relative to the hcp structure. The difference in energy had a negative value for all pressures between 0 and $600 \mathrm{GPa}$, which indicates that the fcc structure was stable. These results are consistent with 
previous calculations [22]. The stability of the fcc structure at high temperatures was not investigated in this study. A phase transformation from the fcc to the hcp structure has not been observed at ambient pressure in previous experimental studies, and the difference in enthalpy calculated in this study increased as the pressure increased. These data imply that the fcc structure is stable at high pressures and high temperatures.

\section{Equation of state for $P t$}

The $P-V-T$ data were calculated to $300 \mathrm{GPa}$ and $3000 \mathrm{~K}$ using the AIMD method. We determined the thermoelastic parameters of Pt at ambient temperature, $300 \mathrm{~K}$, using the third-order Birch-Murnaghan equation of state. The Birch-Murnaghan EOS is given by following expression:

$P_{s t}=\frac{3}{2} B_{T 0}\left[\left(\frac{V_{0}}{V}\right)^{\frac{7}{3}}-\left(\frac{V_{0}}{V}\right)^{\frac{5}{3}}\right]\left\{1+\frac{3}{4}\left(B_{T}{ }^{\prime}-4\right)\left[\left(\frac{V_{0}}{V}\right)^{\frac{2}{3}}-1\right]\right\}$,

where $B_{T 0}$ is the isothermal bulk modulus at $300 \mathrm{~K}, V_{0}$ is the zero-pressure volume, $V$ is the high-pressure volume, and $B_{T}$ ' is the pressure derivative of $B_{T 0}$. 
The thermal pressure, $P_{t h}$, was evaluated in two ways in this study. Both the thermal pressure EOS and the Mie-Grüneisen-Debye EOS were used [32]. The thermal pressure, $P_{t h}$, of the thermal pressure EOS can be written as follows:

$$
P_{t h}=\alpha_{0} B_{T 0}\left(T-T_{300}\right)+\left(\frac{\partial B_{T}}{\partial T}\right)_{V}\left(T-T_{300}\right) \ln \left(\frac{V_{0}}{V}\right)+\left(\frac{\partial^{2} P}{\partial T^{2}}\right)_{V}\left(T-T_{300}\right)^{2} .
$$

The fitting parameters of the Birch-Murnaghan EOS combined with the thermal pressure EOS are $V_{0}, B_{T 0}, B_{T}, \alpha_{0},\left(\frac{\partial B_{T}}{\partial T}\right)_{V}$, and $\left(\frac{\partial^{2} P}{\partial T^{2}}\right)_{V}$. The thermal pressure, $P_{t h}$, of the Mie-Grüneisen-Debye EOS can be written as follows:

$$
P_{t h}=\frac{\gamma}{V}\left[E_{t h}(V, T)-E_{t h}\left(V, T_{0}\right)\right]
$$

where $\gamma$ is the Grüneisen parameter and $E_{t h}$ is the thermal energy. The thermal energy is calculated from the Debye model as follows:

$$
E_{t h}=\frac{9 n R T}{\left(\frac{\theta}{T}\right)^{3}} \int_{0}^{\frac{\theta}{T}} \frac{\xi^{3}}{e^{\xi}-1} d \xi,
$$

where $n$ is the number of atoms in a formula unit, $\mathrm{R}$ is the universal gas constant, and $\theta$ is the Debye temperature. The dependence of the volume on the Debye temperature and the Grüneisen parameter is described by the following 
equations:

$$
\begin{aligned}
& \theta=\theta_{0} \exp \left[\frac{\gamma-\gamma_{0}}{q}\right], \\
& \gamma=\gamma_{0}\left(\frac{V}{V_{0}}\right)^{q}
\end{aligned}
$$

where $\gamma_{0}$ is the Grüneisen parameter at zero pressure, and $q$ is a dimensionless parameter. As the measured Debye temperature $\theta_{0}$ was around $240 \mathrm{~K}$ [35], this value was fixed in our calculations. The fitting parameters of the Birch-Murnaghan EOS combined with the Mie-Grüneisen-Debye EOS are $V_{0}$, $B_{T 0}, B_{T}^{\prime}, \gamma_{0}$, and $q$.

A least squares fit of the compression data at $300 \mathrm{~K}$ yielded $V_{0}=$ 15.092 $\AA^{3}, B_{T 0}=294.9 \mathrm{GPa}$, and $B_{T}{ }^{\prime}=5.05$ using the pressure correction of $\Delta P$ $=5.485 \mathrm{GPa}$. Our results are consistent with the recent experimental values of $V_{0}=15.095 \AA^{3}, B_{T 0}=273.6 \mathrm{GPa}$, and $B_{T}{ }^{\prime}=5.23[36]$.

The results of the fit of our $P-V-T$ data to the thermal pressure EOS and the Mie-Grüneisen-Debye EOS are summarized in Table 2. The values of $V_{0}$, $B_{T 0}$ and $B_{T}$ ' from the thermal pressure EOS are in very good agreement with the analysis using the Mie-Grüneisen-Debye EOS. Fig. 4 shows the thermal pressure, 
$P_{t h}$, of Pt versus the cell volume. It can be seen that the thermal pressure decreases as the cell volume decreases. The change in thermal pressure under compression is smaller than the change in thermal pressure with temperature. The pressure dependence of the thermal pressure calculated in this study is in agreement with that reported in previous studies $[15,37,38]$. However, some studies have reported an opposite pressure dependence to the findings of our study [16,39]. One possible reason for the inconsistency is the uncertainty of high-pressure experiments. The both conflicted EOSs for Pt [16,39] were determined by the same pressure scale of $\mathrm{MgO}$ [40]. As some types of EOS for $\mathrm{MgO}$ have been reported by many previous studies, the pressure scale of $\mathrm{MgO}$ [40] used in the conflicted studies needs to be further verified to minimize the uncertainty of the EOS for Pt. The thermal parameters of the thermal pressure EOS and the Mie-Grüneisen-Debye EOS were determined to be $\alpha_{0}=1.94(7) \times 10^{-5}\left(\mathrm{~K}^{-1}\right),\left(\partial B_{T} / \partial T\right)_{V}=-0.0038(5)\left(\mathrm{GPa} \mathrm{K} \mathrm{K}^{-1}\right),\left(\partial^{2} P / \partial T^{2}\right)_{V}=$ $1.7(15) \times 10^{-7}\left(\mathrm{GPa}^{2} \mathrm{~K}^{-2}\right), \gamma_{0}=2.18(4)$, and $q=1.75(9)$.

Fig. 5a shows a comparison of the Hugoniot curve between the experimental data and our calculations. Fig. 5b also shows the fitted isothermal compressibility curves at $300,1000,2000$, and $3000 \mathrm{~K}$ compared with experimental data at ambient 
temperature [26]. Both figures indicate that a small overestimation of the calculated volume relative to the experimental data was confirmed. Table 3 lists the pressure at selected compressions and temperatures based on the EOS used in this study. Fig. 6 shows the calculated thermal expansion coefficient. As the pressure increases, the dependence of the pressure on the thermal expansion decreases at temperatures above the Debye temperature. This is in good agreement with the typical properties of condensed materials.

The thermodynamic properties of Pt have been measured at ambient pressure. The observed Grüneisen parameters $[35,41]$ were in the range 1.81-2.92, which is in general agreement with the value of 2.18 in our calculations. Fig. 7 shows a comparison between experimental data and our calculations of the bulk modulus and thermal expansion at ambient pressure. The bulk modulus determined in this study was underestimated by $\sim 3 \%$ compared with the experimental values at temperatures in the range $300-1400 \mathrm{~K}$. In contrast, the thermal expansion was overestimated in our calculations compared with the experimental values. The discrepancy in the thermal expansion increased as temperature increased. 
Although previous workers have tried to establish internally consistent pressure EOSs using experimental data from shock-wave, ultrasonic, X-ray, and thermochemical methods, the internal consistency of the EOSs for several solids remains an open question. Recently, more reliable EOS of Pt than that from previous studies was proposed $[15,16]$. The pressure derivative determined in our study is in good agreement with that reported by previous studies (Tables 1 and 2), but the bulk modulus from our study is slightly higher than that from previous experimental studies. We used the simple constant shift in pressure $(\Delta P)$ to correct for the systematic error in our calculations. The corrected EOS is in better agreement with the experimental values than is the uncorrected EOS. However, a small discrepancy remains. This is likely to be caused by the uncertainties in the potential of $\mathrm{Pt}$ and the approximation used in our calculations (LDA). In contrast, it is known that the uncertainty in the experimental data is significant, especially at high temperatures, because of the errors in temperature measurements in high-pressure experiments. This indicates that the experimental data do not have an indisputable advantage at high temperatures compared with the AIMD simulations. Therefore, we did not use a simple pressure correction for high-temperature conditions. 


\section{Anharmonic effect}

The Grüneisen parameter only depends on the volume in the quasiharmonic approximation for a classical solid. However, the actual Grüneisen parameter has a temperature dependence because of intrinsic anharmonic effects. Therefore, we investigated these temperature-dependent anharmonic effects. The total pressure can be written as the sum of the static, quasiharmonic, and anharmonic terms, as:

$P(V, T)=P_{s t}(V)+P_{q}(V, T)+P_{a}(V, T)$.

The quasiharmonic part of Eq. (8) can be written as:

$P_{q}(V, T)=\frac{E_{v i b}^{q}}{V} \gamma_{q}(V)$

where $E_{v i b}^{q}$ and $\gamma_{q}(V)$ are the quasiharmonic vibrational energy and the quasiharmonic Grüneisen parameter, respectively.

At high temperatures, the anharmonic part is important and the anharmonic free energy can be described by a quadratic function of the temperature: 
$F_{a}(V, T)=\frac{3}{2} N k_{B} a(V) T^{2}$,

where $k_{B}$ and $N$ are the Boltzmann constant and the number of atoms in the calculated cell, respectively, and $a(V)$ is the intrinsic anharmonic parameter, which is related to the change in temperature of the phonon spectrum. The anharmonic part of Eq. (8) can be written as follows:

$P_{a}(V, T)=-\left(\frac{\partial F}{\partial V}\right)_{T}=-\frac{3}{2} N k_{B} \frac{d \ln a}{d \ln V} a(V) \frac{T^{2}}{V} .$.

In the case of a classical approximation, such as the Debye model, the quasiharmonic vibrational energy at high temperatures is given by:

$E_{v i b}^{q}=3 N k_{B} T$.

Therefore, Eq. (8) can finally be rewritten as follows:

$P(V, T)=P_{s t}(V)+\frac{3 N k_{B} T}{V}\left[\gamma_{q}(V)-\frac{1}{2} a(V) \frac{d \ln a}{d \ln V} T\right]$.

The $3 N$ term denotes the number of vibrational degrees of freedom per $N$ atoms.

In molecular dynamic simulations, the number of degrees of freedom is decreased to $3 N-3$. The effective Grüneisen parameter, including the intrinsic anharmonic effects, can be written as follows: 
$\gamma_{e}(V, T)=\gamma_{q}(V)-\frac{1}{2} a(V) \frac{d \ln a}{d \ln V} T$.

Fig. 8 shows the temperature dependence of the effective Grüneisen parameter due to the intrinsic anharmonic effects. The effective Grüneisen parameter was calculated using the pressure-volume data at each temperature. The difference in the effective Grüneisen parameter at different temperatures is small at high temperatures. The temperature dependence decreased as the volume decreased (or as the pressure increased). This indicates that the anharmonic effect on the thermal pressure is not significant at high pressures, which agrees with the observation that the pressure dependence of the thermal pressure decreases as the pressure increases, as shown in Fig. 4.

\section{Conclusions}

We have determined the $P-V-T$ equation of state for platinum based on non-empirical calculations within the framework of AIMD simulations using all-electron PAW methods, except for the use of a simple pressure correction $(\Delta P)$. The intrinsic anharmonic effects in platinum were found to be small in the temperature range studied. We confirmed that a combination of the LDA and AIMD methods 
leads to an accurate prediction of the EOS of platinum (once the systematic error in $\mathrm{P}$ associated with LDA is corrected for). From ground state calculations, we have confirmed the stability of the fcc structure in the pressure range investigated. The calculated EOS is in better agreement with the measured values than with those of previous calculations. However, a small discrepancy between our EOS and the measured values was confirmed at high pressures. This discrepancy is likely to be caused by uncertainties both in our simulations and in previous experiments.

\section{Acknowledgments}

This work made use of the UCL research computing facility and of the JAMSTEC computing facility. This work was supported by NERC Computational Mineral Physics Consortium, UK and Grant-in-Aid for Scientific Research from the Ministry of Education, Culture, Sports, Science and Technology, Japan. 


\section{References}

[1] F. Datchi, R. LeToullec, P. Loubeyre, J. Appl. Phys. 81 (1997) 3333-3339.

[2] W.B. Holzapfel, J. Appl. Phys., 93 (2003) 1813-1818.

[3] O.L. Anderson, D.G. Isaak, S. Yamamoto, J. Appl. Phys. 65 (1989) 1534-1543.

[4] J.M. Brown, J. Appl. Phys. 86 (1999) 5801-5808.

[5] S. Ono, J.P. Brodholt, D. Alfè, M. Alfredsson, G.D. Price, J. Appl. Phys. 86 (2007) 5801-.5808

[6] M. Matsui, S.C. Parker, M. Leslie, Am. Mineral. 85 (2000) 312-316.

[7] S. Speziale, C.-S. Zha, T.S. Duffy, R.J. Hemley, H.-K. Mao, J. Geophys. Res. 106 (2001) 515-528.

[8] J.C. Jamieson, J.N. Fritz, M.H. Manghnani, in: S. Akimoto, M.H. Manghnani (Eds.), High-Pressure Research in Geophysics, Center for Academic Publishing, Tokyo, 1982, pp. 27-48.

[9] N.C. Holmes, J.A. Moriarty, G.R. Gathers, W.J. Nellis, J. Appl. Phys. 66 (1989) 2962-2967.

[10] G. Fiquet, D. Andrault, A. Dewaele, T. Charpin, M. Kunz, D. Häusermann, Phys. Earth Planet. Inter. 105 (1998) 21-31. 
[11] S.-H. Shim, T.S. Duffy, G. Shen, Phys. Earth Planet. Inter. 120 (2000) 327-338.

[12] A.R. Oganov, S. Ono, Nature 430 (2004) 445-448.

[13] S-H. Shim, T.S. Duffy, G. Shen, Nature 411 (2001) 571-574.

[14] L. Chudinovskikh, R. Boehler, Nature 411 (2001) 574-577.

[15] P.I. Dorogokupets, A.R. Oganov, Phys. Rev. B 75 (2007) 024115.

[16] Y. Fei, A. Ricolleau, M. Frank, K. Mibe, G. Shen, V. Prakapenka, Proc. Natl. Acad. Sci. 104 (2007) 9182-9186.

[17] W.B. Holzapfel, M.F. Nicol, High Press. Res. 27 (2007) 377-392.

[18] K. Takemura, High Press. Res. 27 (2007) 465-472.

[19] F.D. Stacey, P.M. Davis, Phys. Earth Planet. Int. 142 (2004) 137-184.

[20] C. Bercegeay, S. Bernard, Phys. Rev. B 72 (2005) 214101.

[21] S. Xiang, L. Cai, Y. Bi, F. Jing, S. Wang, Phys. Rev. B 72 (2005) 184102.

[22] E. Menéndez-Proupin, A. K. Singh, Phys. Rev. B 76 (2007) 054117.

[23] T. Sun, K. Umemoto, Z. Wu, J.C. Zheng, R.M. Wentzcovitch, Phys. Rev.

B 78 (2008) 024304.

[24] P.E. Blöchl, Phys. Rev. B 50 (1994) 17953-17979.

[25] G. Kresse, J. Furthmüller, Phys. Rev. B 54 (1996) 11169-11186. 
[26] G. Kresse, D. Joubert, Phys. Rev. B 59 (1999) 1758-1775.

[27] Y. Wang, J.P. Perdew, Phys. Rev. B 44 (1991) 13298-13307.

[28] J.P. Perdew, K. Burke, M. Ernzerhof, Phys. Rev. Lett. 77 (1996) 3865-3868.

[29] S. Nosé, Molec. Phys., 52 (1984) 255-268.

[30] S. Taioli, C. Cazorla, M.J. Gillan, D. Alfè, Phys. Rev. B 75 (2007) 214103.

[31] S. Ono, Int. J. Mol. Sci. 10 (2009) 4342-4351.

[32] M.J. Gillan, D. Alfè, J.P. Brodholt, L. Vočadlo, G.D. Price, Rep. Prog. Phys. 69 (2006) 2365-2441.

[33] A.R. Oganov, J.P. Brodholt, G.D. Price, Earth Planet. Sci. Lett. 184 (2001) $555-560$.

[34] I. Jackson, S.M. Rigden, Phys. Earth Planet. Int. 96 (1996) 85-112.

[35] K.A. Gschneidner, Solid State Phys. 16 (1964) 275-426.

[36] A. Dewaele, P. Loubeyre, M. Mezouar, Phys. Rev. B 70 (2004) 094112.

[37] M. Matsui, E. Ito, T. Katsura, D. Yamazaki, T. Yoshino, A. Yokoyama, K. Funakoshi, J. Appl. Phys. 105 (2009) 013505.

[38] M. Yokoo, N. Kawai, K.G. Nakamura, K. Kondo, Y. Tange, T. Tsuchiya, Phys. 
Rev. B 80 (2009) 104115.

[39] C.S. Zha, K. Mibe, W.A. Bassett, O. Tschauner, H..K. Mao, R.J. Hemley, J. Appl. Phys. 103 (2008) 054908.

[40] S. Speziale, C.S. Zha, T.S. Duffy, R.J. Hemley, H.K. Mao, J. Geophys. Res. 106 (2001) 515-528.

[41] M.W. Guinan, D.J. Steinberg, J. Phys. Chem. Solid 35 (1974) 1501-1512.

[42] P.I. Dorogokupets, A. Dewaele, High Press. Res. 27 (2007) 431-446.

[43] T. Tsuchiya, K. Kawamura, Phys. Rev. B 66 (2002) 094115.

[44] S.M. Collard, R.B. McLellan, Acta Metall. Mater. 40 (1992) 699-702.

[45] Y.S. Touloukian, R.K. Kirby, R.E. Taylar, P.D. Desai, Thermophysical properties of matter, IFI/Plenum, New York, 1977. 
Table 1.

Bulk modulus of Pt at $0 \mathrm{~K}$. The Birch-Murnaghan equation of state was used to calculate the bulk moduli of fcc-structured Pt. Key: $B_{0}=$ isothermal bulk modulus, $B_{0}$, $=$ first pressure deviation of bulk modulus, $V_{0}=$ volume at zero pressure from our calculations, $\mathrm{LDA}=$ local density approximation, GGA = generalized gradient approximation, $\mathrm{SO}=$ with spin-orbit coupling, $\mathrm{PC}=$ with pressure correction, $\mathrm{PW} 91=$ GGA functional from Wang and Perdew [27], PBE96 = GGA functional from Perdew et al [28]. Experimental values were measured at $\sim 300 \mathrm{~K}$.

\begin{tabular}{llll}
\hline$B_{0}(\mathrm{GPa})$ & $B_{0}{ }^{\prime}$ & $V_{0}\left(\AA^{3}\right)$ & \\
\hline 273.6 & 5.23 & 15.10 & High-P experiment [36] \\
273.5 & 4.70 & 15.10 & High-P experiment [39] \\
276.4 & 5.12 & 15.07 & High-P experiment [38] \\
276.07 & 5.30 & 15.10 & Empirical EOS [15] form experimental data \\
277 & 4.95 & 15.10 & Empirical EOS [16] form experimental data \\
277.3 & 5.12 & 15.10 & Empirical EOS [42] form experimental data \\
273 & 5.20 & 15.10 & Empirical EOS [37] form experimental data \\
280.8 & 6.2 & 14.9 & LDA+SO [43] \\
305.99 & & 14.81 & LDA [20] \\
297.01 & & 14.92 & LDA+SO [20] \\
250.85 & & 15.59 & GGA [20] \\
241.81 & & 15.74 & GGA+SO [20] \\
\hline
\end{tabular}


Table 1. (continued).

\begin{tabular}{llll}
\hline$B_{0}(\mathrm{GPa})$ & $B_{0}{ }^{\prime}$ & $V_{0}\left(\AA^{3}\right)$ & \\
\hline 300.9 & 5.814 & 14.90 & LDA [21] \\
243.3 & 5.866 & 15.77 & GGA [21] \\
301.17 & 5.533 & 14.784 & LDA+SO [23] \\
310.24 & 5.126 & 14.888 & LDA (this study) \\
289.28 & 5.193 & 15.095 & LDA+PC (this study) \\
305.20 & 5.139 & 14.921 & LDA+SO (this study) \\
287.84 & 5.195 & 15.095 & LDA+SO+PC (this study) \\
251.21 & 5.207 & 15.804 & GGA(PW91) (this study) \\
316.35 & 4.991 & 15.095 & GGA(PW91)+PC (this study) \\
254.17 & 5.221 & 15.701 & GGA(PBE96) (this study) \\
310.11 & 5.033 & 15.095 & GGA(PBE96)+PC (this study) \\
\hline
\end{tabular}


Table 2.

Thermoelastic parameters of $\mathrm{Pt}$ using the thermal pressure EOS and the Mie-Grüneisen-Debye EOS. Numbers between parentheses are the errors of the least squares fitting.

\begin{tabular}{ll}
\hline TP EOS & \\
\hline$V_{0} \quad\left(\AA^{3}\right)$ & $15.105(11)$ \\
$B_{T 0} \quad(\mathrm{GPa})$ & $290.8(21)$ \\
$B_{T}{ }^{\prime}$ & $5.11(2)$ \\
$\alpha_{0}\left(10^{-5} \mathrm{~K}^{-1}\right)$ & $1.94(7)$ \\
$\left(\partial B_{T} / \partial T\right)_{V}\left(\mathrm{GPa} \mathrm{K}^{-1}\right)$ & $-0.0038(5)$ \\
$\left(\partial^{2} P / \partial T^{2}\right)_{V}\left(10^{-7} \mathrm{GPa}^{2} \mathrm{~K}^{-2}\right)$ & $1.7(15)$ \\
\hline $\mathrm{MGD} \mathrm{EOS}$ & $15.098(10)$ \\
\hline$V_{0}\left(\AA^{3}\right)$ & $292.0(21)$ \\
$B_{T 0}(\mathrm{GPa})$ & $5.10(2)$ \\
$B_{T}{ }^{\prime}$ & $2.18(4)$ \\
$\gamma_{0}$ & $1.75(9)$ \\
\hline
\end{tabular}


Table 3.

Pressure-Temperature-Volume table for Pt from this study. The unit of pressure is GPa.

\begin{tabular}{rrrrrrrr}
\hline $1-V / V_{0}$ & $300 \mathrm{~K}$ & $500 \mathrm{~K}$ & $1000 \mathrm{~K}$ & $1500 \mathrm{~K}$ & $2000 \mathrm{~K}$ & $2500 \mathrm{~K}$ & $3000 \mathrm{~K}$ \\
\hline 0.00 & 0.00 & 1.10 & 4.03 & 7.01 & 10.08 & 13.23 & 16.47 \\
0.01 & 3.00 & 4.09 & 7.00 & 9.97 & 13.02 & 16.15 & 19.37 \\
0.02 & 6.19 & 7.27 & 10.16 & 13.11 & 16.14 & 19.25 & 22.45 \\
0.03 & 9.57 & 10.65 & 13.53 & 16.45 & 19.46 & 22.55 & 25.73 \\
0.04 & 13.18 & 14.24 & 17.10 & 20.01 & 23.00 & 26.07 & 29.23 \\
0.05 & 17.01 & 18.06 & 20.90 & 23.79 & 26.76 & 29.81 & 32.95 \\
0.06 & 21.08 & 22.13 & 24.95 & 27.81 & 30.76 & 33.80 & 36.92 \\
0.07 & 25.41 & 26.45 & 29.25 & 32.09 & 35.02 & 38.04 & 41.14 \\
0.08 & 30.01 & 31.05 & 33.83 & 36.65 & 39.56 & 42.55 & 45.63 \\
0.09 & 34.91 & 35.94 & 38.70 & 41.50 & 44.39 & 47.36 & 50.42 \\
0.10 & 40.13 & 41.14 & 43.88 & 46.66 & 49.53 & 52.48 & 55.52 \\
0.11 & 45.67 & 46.68 & 49.39 & 52.15 & 55.00 & 57.93 & 60.95 \\
0.12 & 51.57 & 52.57 & 55.27 & 58.01 & 60.83 & 63.74 & 66.73 \\
0.13 & 57.86 & 58.85 & 61.52 & 64.24 & 67.04 & 69.93 & 72.90 \\
0.14 & 64.55 & 65.53 & 68.18 & 70.88 & 73.66 & 76.52 & 79.47 \\
0.15 & 71.68 & 72.65 & 75.28 & 77.95 & 80.71 & 83.55 & 86.48 \\
\hline & 79.27 & 80.24 & 82.84 & 85.49 & 88.23 & 91.05 & 93.96 \\
\hline & & & & & & & \\
\hline
\end{tabular}


Table 3. (continued).

\begin{tabular}{|c|c|c|c|c|c|c|c|}
\hline 0.17 & 87.37 & 88.33 & 90.91 & 93.54 & 96.25 & 99.05 & 101.93 \\
\hline 0.18 & 96.00 & 96.95 & 99.51 & 102.11 & 104.80 & 107.58 & 110.44 \\
\hline 0.19 & 105.21 & 106.15 & 108.68 & 111.26 & 113.93 & 116.68 & 119.52 \\
\hline 0.20 & 115.04 & 115.96 & 118.47 & 121.03 & 123.68 & 126.40 & 129.22 \\
\hline 0.21 & 125.52 & 126.44 & 128.93 & 131.46 & 134.08 & 136.79 & 139.58 \\
\hline 0.22 & 136.73 & 137.63 & 140.10 & 142.61 & 145.20 & 147.88 & 150.65 \\
\hline 0.23 & 148.69 & 149.59 & 152.03 & 154.52 & 157.09 & 159.74 & 162.48 \\
\hline 0.24 & 161.49 & 162.37 & 164.79 & 167.25 & 169.80 & 172.43 & 175.14 \\
\hline 0.25 & 175.17 & 176.05 & 178.44 & 180.87 & 183.39 & 186.00 & 188.69 \\
\hline 0.26 & 189.81 & 190.68 & 193.04 & 195.45 & 197.95 & 200.53 & 203.19 \\
\hline 0.27 & 205.49 & 206.34 & 208.68 & 211.07 & 213.54 & 216.09 & 218.73 \\
\hline 0.28 & 222.28 & 223.13 & 225.44 & 227.80 & 230.24 & 232.77 & 235.38 \\
\hline 0.29 & 240.28 & 241.12 & 243.40 & 245.73 & 248.15 & 250.65 & 253.24 \\
\hline 0.30 & 259.59 & 260.41 & 262.67 & 264.98 & 267.37 & 269.84 & 272.40 \\
\hline 0.31 & 280.31 & 281.13 & 283.36 & 285.64 & 288.00 & 290.45 & 292.98 \\
\hline 0.32 & 302.57 & 303.37 & 305.58 & 307.83 & 310.16 & 312.58 & 315.09 \\
\hline 0.33 & 326.49 & 327.28 & 329.46 & 331.68 & 333.99 & 336.38 & 338.86 \\
\hline 0.34 & 352.22 & 353.00 & 355.15 & 357.34 & 359.62 & 361.98 & 364.43 \\
\hline 0.35 & 379.92 & 380.69 & 382.81 & 384.97 & 387.22 & 389.55 & 391.97 \\
\hline 0.36 & 409.76 & 410.51 & 412.60 & 414.74 & 416.96 & 419.26 & 421.65 \\
\hline
\end{tabular}




\section{Figure captions}

Fig. 1.

Pressure versus volume data obtained from DFT calculations for Pt. a, The open triangles and crosses denote the volumes from the LDA calculations without, and with spin-orbit coupling, respectively. The zero pressure equilibrium volume, $V_{0}$, bulk modulus, $B_{0}$, and first pressure deviation of bulk modulus, $B_{0}$ ', without, and with spin-orbit coupling are $14.888 \AA^{3}, 310.24 \mathrm{GPa}$, and 5.126 for calculations without spin-orbit coupling, and $14.921 \AA^{3}, 305.20 \mathrm{GPa}$, and 5.139 for calculations with spin-orbit coupling. b, The difference in volume without and with spin-orbit coupling. The solid squares denote the difference in volume with spin-orbit coupling relative to the difference in volume without it.

\section{Fig. 2.}

Difference in the thermal pressures of GGA exchange-correlation functionals relative to LDA. The thermal pressure was calculated using the AIMD method at 0 and 300 GPa. PW91 and PBE 96 denote GGAs from Wang and Perdew [27] and Perdew et al. [28], respectively.

\section{Fig. 3.}

Difference in enthalpy between the fcc and hcp structures. The solid lines denote the difference in enthalpy of the fcc structure relative to the hcp structure as a function of pressure using the LDA formulation.

Fig. 4. 
A plot of the thermal pressure $\left(P_{t h}\right)$ versus cell volume for Pt. The solid circles denote the calculated thermal pressure up to $3000 \mathrm{~K}$.

\section{Fig. 5.}

a, A hugoniot comparison of the experimental data with our calculations. The solid squares denote data from shock compression experiments [8,9]. The solid line denotes the Hugoniot curve from our calculations. b, Change in isothermal volume of Pt. The solid lines denote the calculated isotherms at 300, 1000, 2000, and $3000 \mathrm{~K}$ (from the bottom to the top) using the AIMD method. The dashed line denotes the isotherm at 0 $\mathrm{K}$ from static calculations. The solid squares denote the experimental data [36].

Fig. 6.

Calculated temperature dependence of the thermal expansion. The results are shown for $0,50,100$, and $300 \mathrm{GPa}$.

\section{Fig. 7}

a, Bulk modulus at ambient pressure. The solid line denotes the experimental data [44]. The solid squares denote the calculated values in this study. b, Thermal linear expansion at ambient pressure. The solid line denotes the regression fit from experimental data [45]. The solid squares denote the calculated values from the EOS for Pt from this study.

Fig. 8 .

Anharmonic effects on the Grüneisen parameter. The solid lines denote the calculated Grüneisen parameter at 1000, 2000, and $3000 \mathrm{~K}$ (from bottom to top) using the AIMD method. 
Ono et al.

Fig. 1

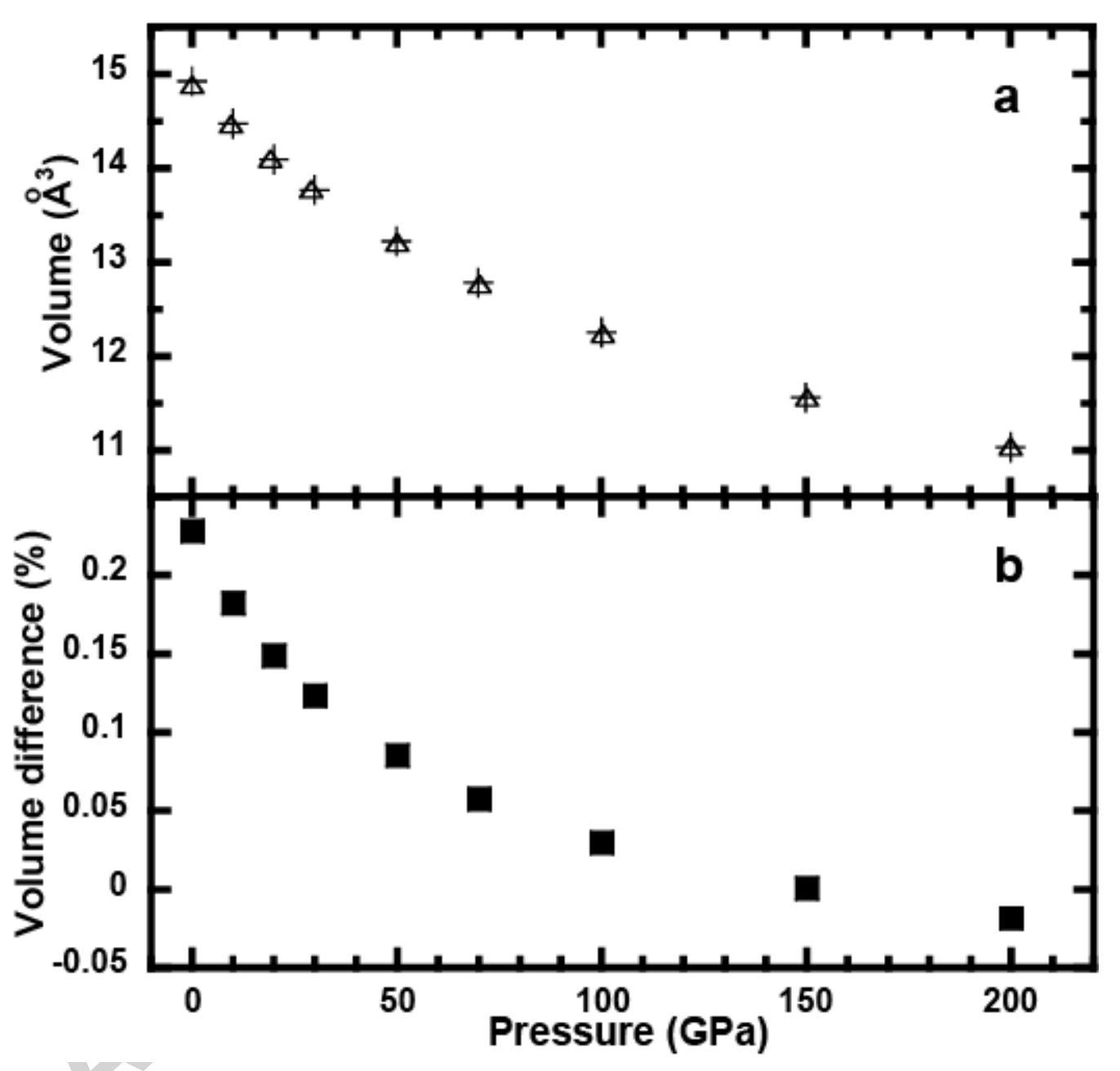


Ono et al.

Fig. 2

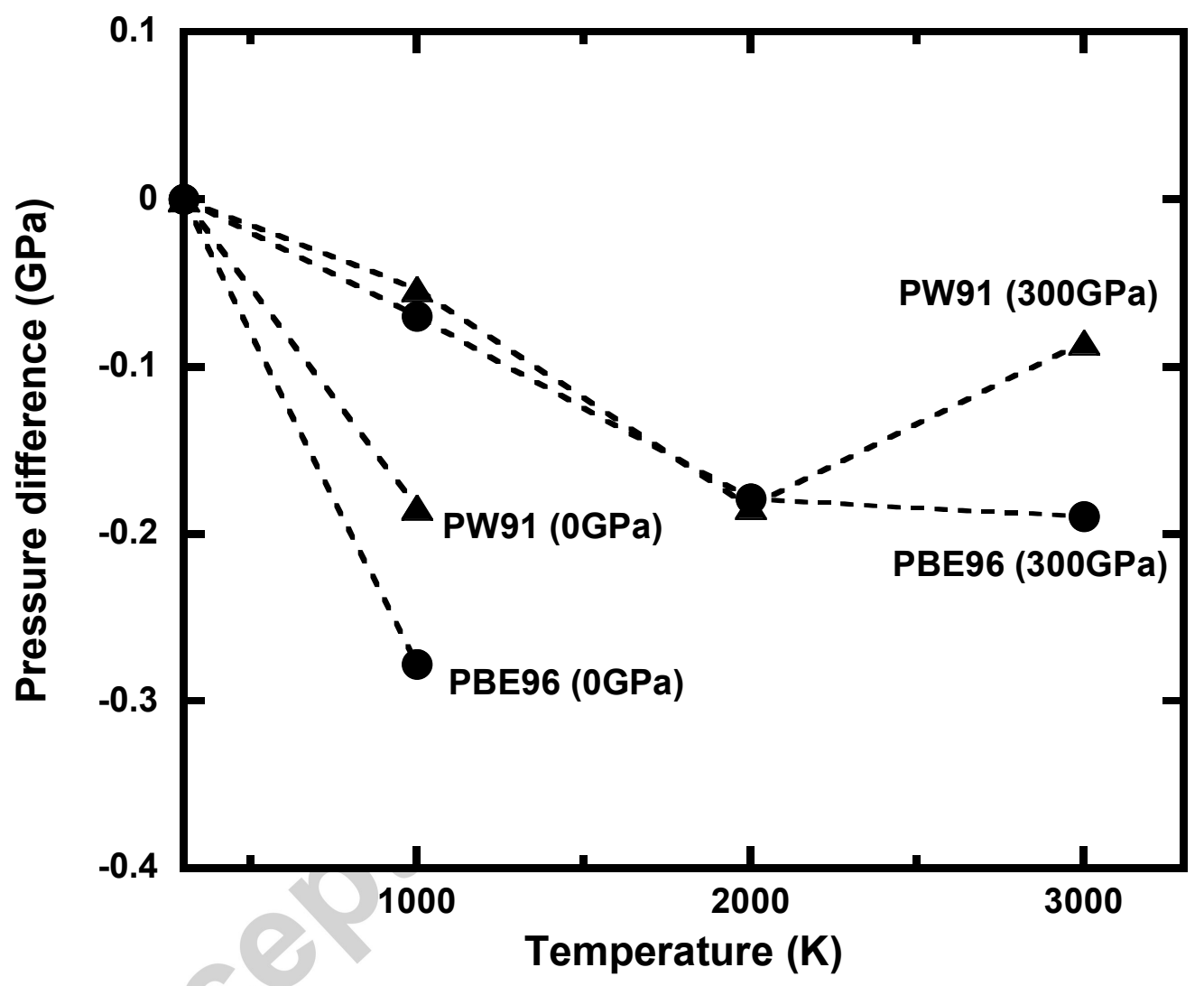


Ono et al.

Fig. 3

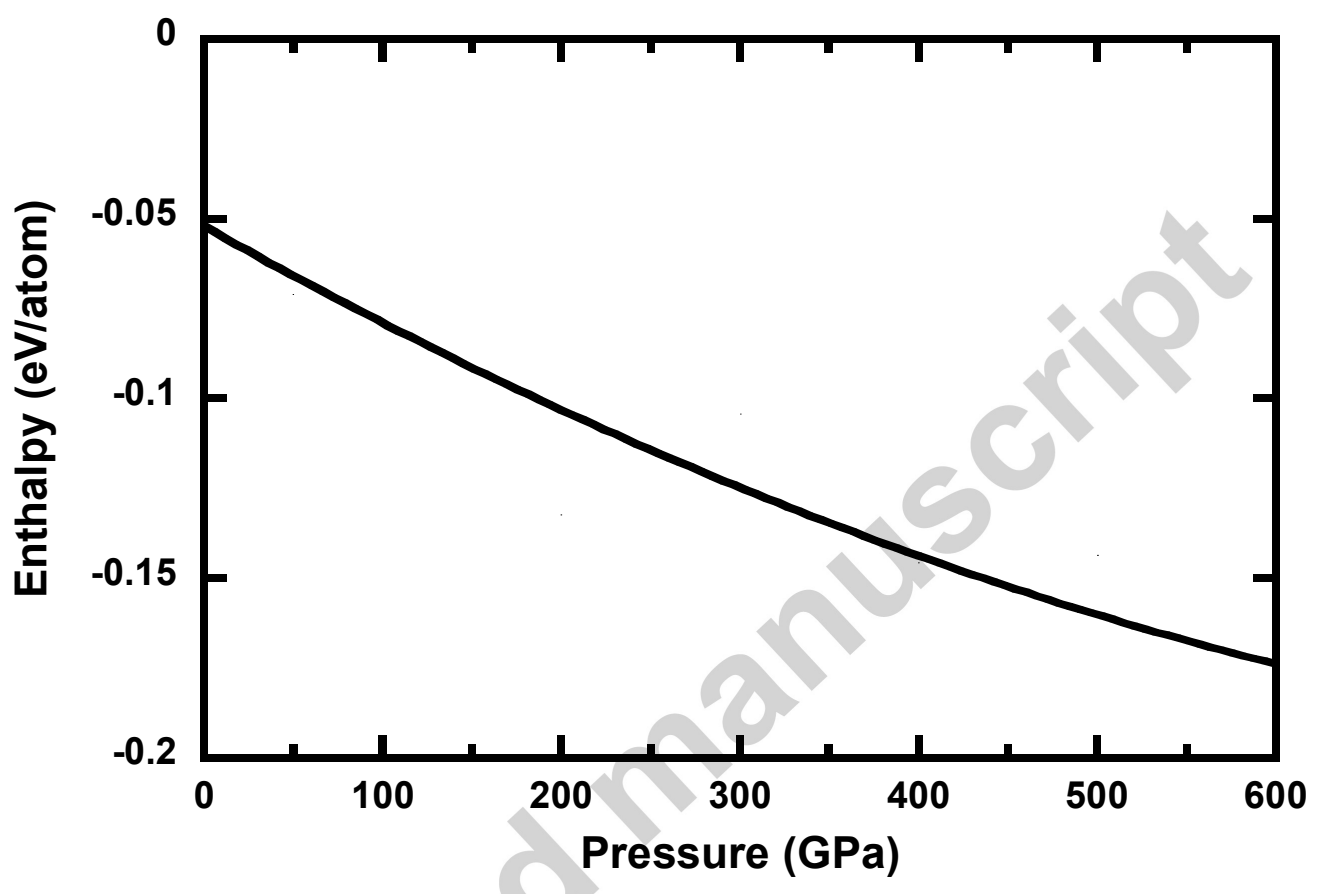


Ono et al.

Fig. 4

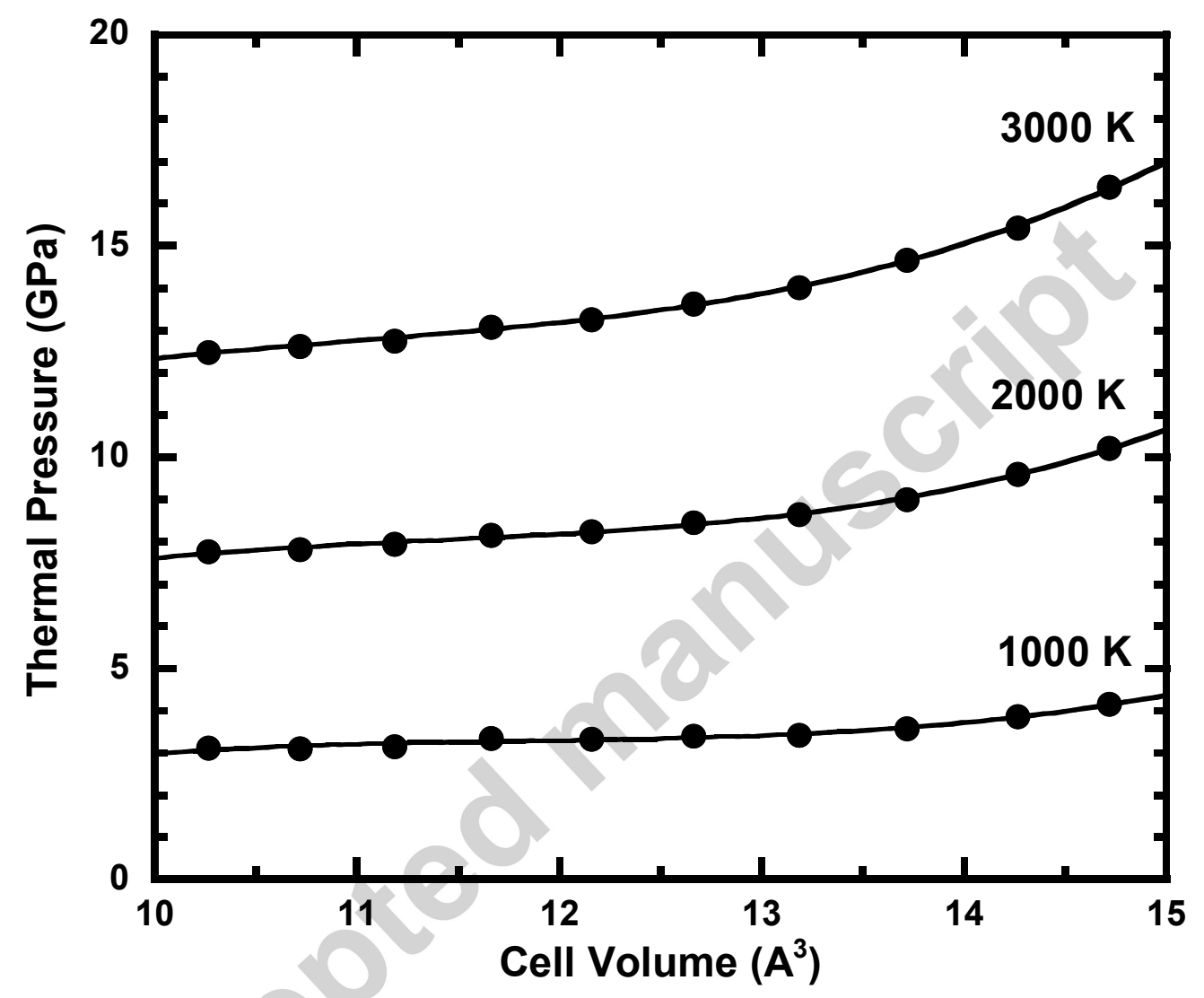


Fig. 5

Ono et al.

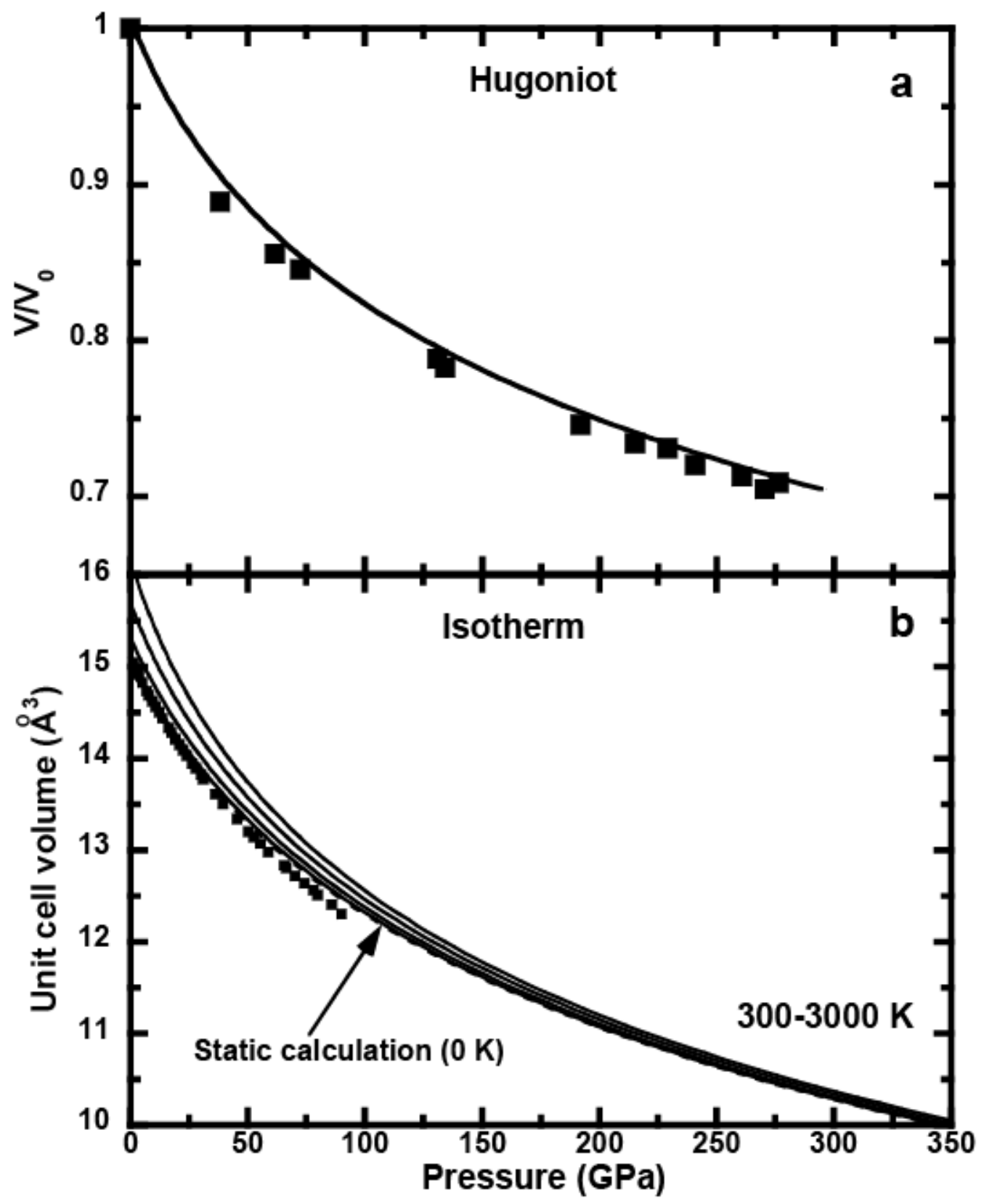


Ono et al.

Fig. 6

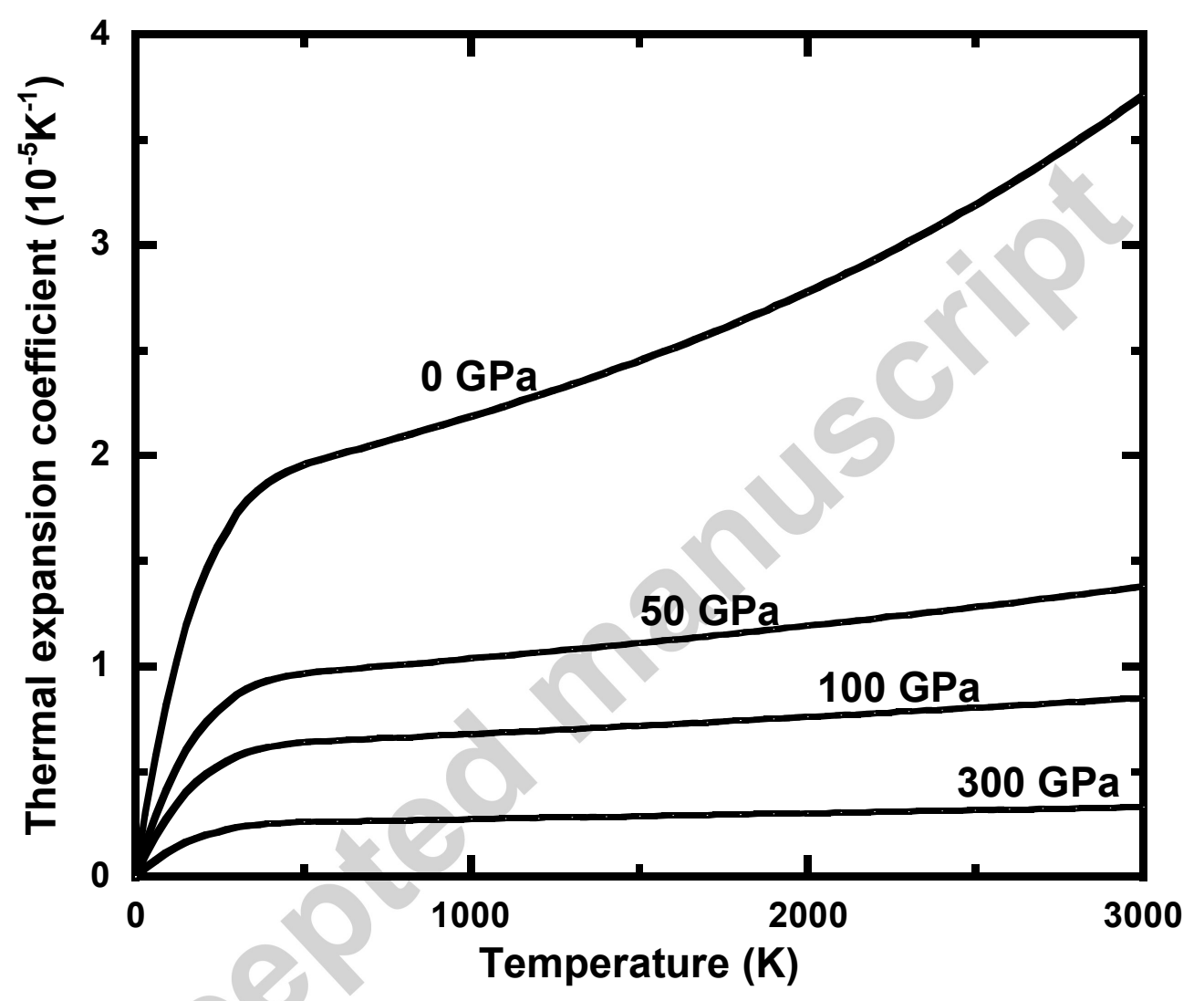


Ono et al.

Fig. 7

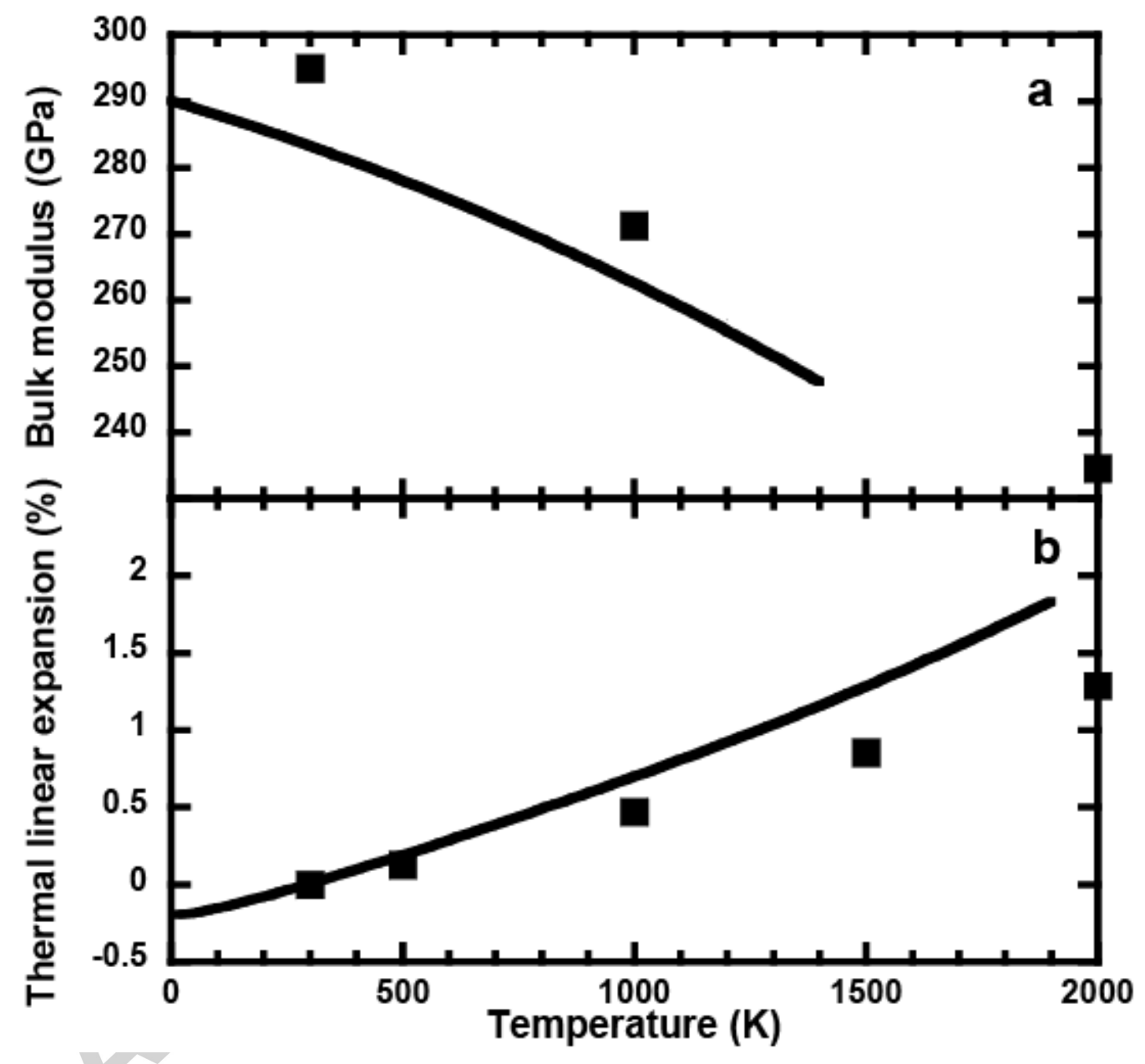


Ono et al.

Fig. 8

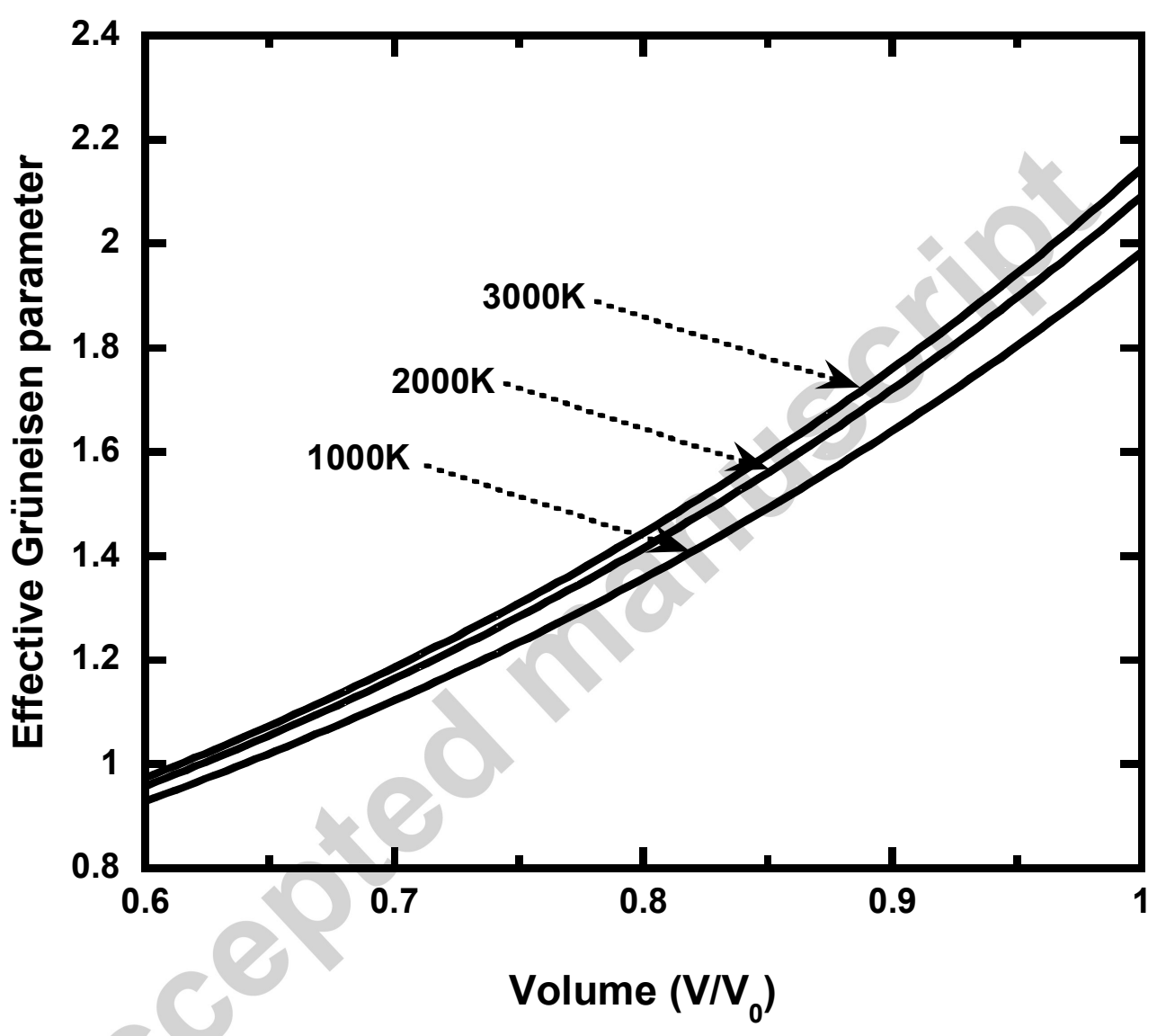


New equation of state for Pt was established.

Pressure and temperature range up to $300 \mathrm{GPa}$ and $3000 \mathrm{~K}$.

Analysis of anharmonicity at high pressures and high temperatures.

First-principles molecular dynamics simulations. 